\title{
Is Blockchain Suitable for Data Freshness? - Age-of-Information Perspective
}

\author{
Sungho Lee, Minsu Kim, Jemin Lee, Member, IEEE, \\ Ruei-Hau Hsu, Member, IEEE, and Tony Q. S. Quek, Fellow, IEEE
}

\begin{abstract}
Recent advances in blockchain have led to a significant interest in developing blockchain-based applications. While data can be retained in blockchains, the stored values can be deleted or updated. From a user viewpoint that searches for the data, it is unclear whether the discovered data from the blockchain storage is relevant for real-time decision-making process for blockchain-based application. The data freshness issue serves as a critical factor especially in dynamic networks handling real-time information. In general, transactions to renew the data require additional processing time inside the blockchain network, which is called ledger-commitment latency. Due to this problem, some users may receive outdated data. As a result, it is important to investigate if blockchain is suitable for providing real-time data services. In this article, we first describe blockchain-enabled (BCE) networks with Hyperledger Fabric (HLF). Then, we define age of information (AoI) of BCE networks and investigate the influential factors in this AoI. Analysis and experiments are conducted to support our proposed framework. Lastly, we conclude by discussing some future challenges.
\end{abstract}

\section{INTRODUCTION}

Ever since Satoshi paved the way for blockchain technology, Bitcoin is considered to be the most popular blockchain-based application, and many researchers have actively studied the underlying architecture sustaining cryptocurrency. One of the outcomes is to utilize the blockchain concept for other nonmonetary applications to ameliorate distributed systems and security issues of some emerging services. For example, an integrated blockchain platform for IoT devices, which are deployed worldwide, is proposed in [1]. The purpose of this platform is to provide the device owners with a practical application that offers comprehensive and immutable log, and allows easy access to their devices without the security concerns that stem from centralized IoT platforms. As another example, a blockchain system for $5 \mathrm{G}$ ultra-dense networks is established to redeem the shortcomings of the existing $4 \mathrm{G}$ authentication [2]. The authentication results are shared among a trusted access point group through the blockchain message propagation mechanism [2].

Unlike stationary networks, there are dynamic networks that are required to handle real-time data. In such cases, data

Corresponding author is $\mathrm{J}$. Lee.

S. Lee, M. Kim, and J. Lee are with Daegu Gyeongbuk Institute of Science and Technology (DGIST), 333, Techno Jungang-daero, Daegu, Republic of Korea 42988 (e-mail: \{seuho2003, kms0603, jmnlee\}edgist.ac.kr).

R. H. Hsu is with National Sun Yat-sen University, 70 Lienhai Rd., Kaohsiung 80424, Taiwan, R.O.C. (e-mail: rhhsu@mail.cse.nsysu.edu.tw).

T. Q. S. Quek is with Information Systems Technology and Design Pillar, Singapore University of Technology and Design, Singapore 487372 (e-mail: tonyquek@sutd.edu.sg). freshness which is an indication of how new or relevant the current retained data is. During decision-making process, a bad decision may be made based on outdated retained data in a dynamic network. For instance, temperature data recorded by heat sensors has to be fed to the central controller in a timely fashion to sense whether there is an outbreak of fire for fire detection. Another example is a vehicular network, where vehicle location information needs to be regularly updated to the control center in order to make useful traffic management.

How do we then estimate the degree of data freshness? Unfortunately, with the traditional performance metrics like delay and throughput, it is difficult to define. To be specific, let us assume that a source generates and transmits data via a channel according to first come first served (FCFS) scheduling. Packets that have not yet delivered will be queued at the source when the channel is busy. In this case, we may be able to achieve higher throughput by transmitting data more frequently. Nevertheless, this may not work well in terms of data freshness because the newly generated data will be backlogged and outdated due to the constrained bandwidth of the system. In an analogous way, although delay can be indeed minimized by transmitting data less frequently, a lower sampling rate results in a lack of update data and makes the data stale [3]. Thus, the traditional performance metrics have limitations on estimating the degree of data freshness. To measure the data freshness degree, the concept of age of information (AoI) has been recently introduced [4][5]. When the generated data at a source (e.g., a sensor) is transmitted to a receiver (e.g., a monitor) for updating the data, the AoI at the receiver is defined as the time elapsed since the generation of the last successfully received update data. Hence, larger AoI means less fresh (i.e., more outdated) information.

Although blockchain can provide more reliable management of data by guaranteeing data integrity, there exists an additional delay to process data. Therefore, it is questionable whether blockchain is suitable for dynamic networks, where sufficiently fresh data is expected. In this article, to explore this unresolved problem, we exploit and analyze AoI for blockchain-enabled (BCE) networks. Specifically, this article aims to 1) investigate influential factors on the AoI in BCE networks, and 2) provide how to design BCE networks for reliable services of AoI-sensitive applications of dynamic networks. 


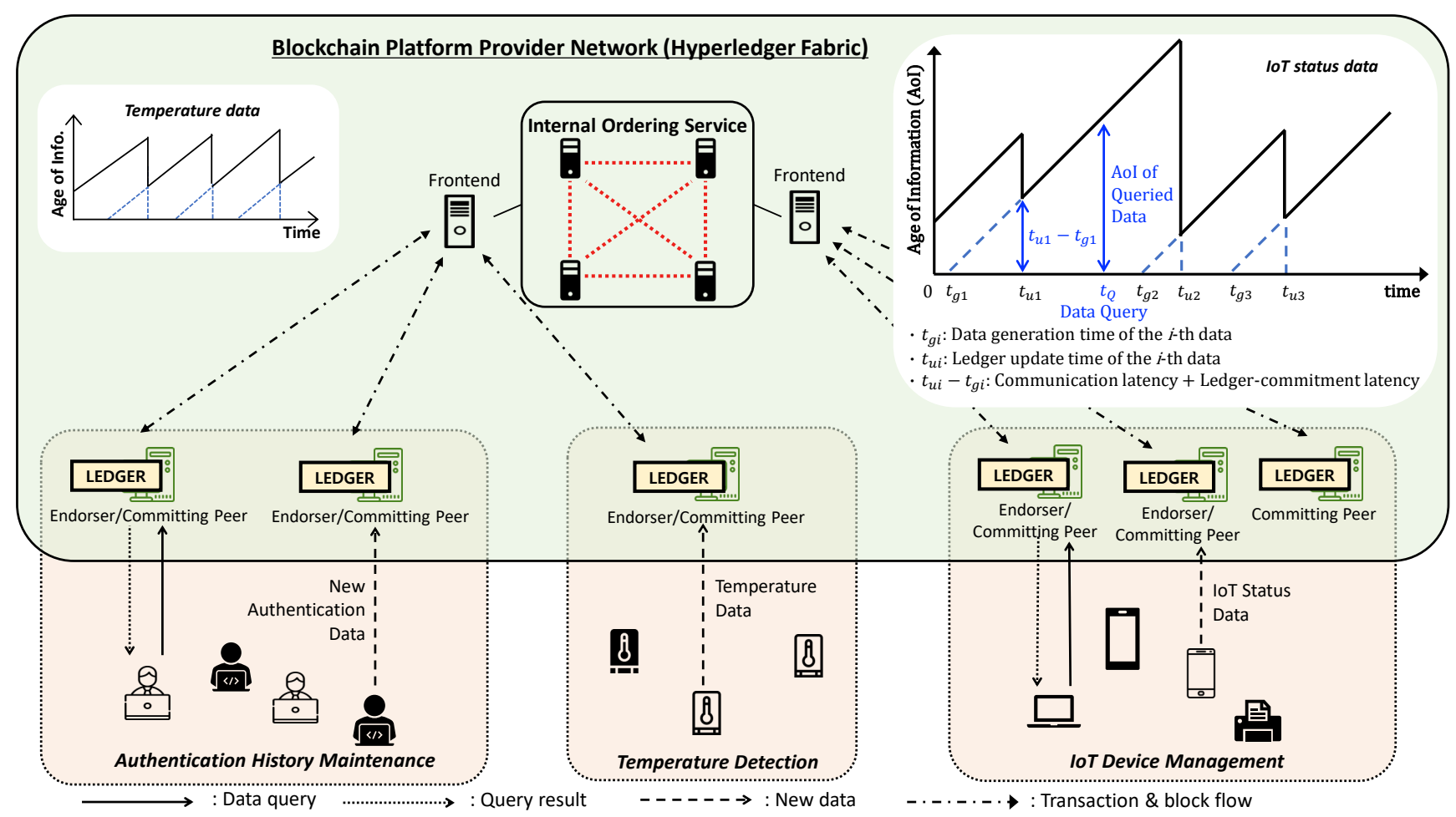

Fig. 1: The structure of a blockchain-enabled network.

\section{BLOCKCHAIN-ENABLED NETWORKS}

\section{A. Private Blockchain}

A public blockchain, as its name indicates, allows any users to involve themselves in the network without requiring authorization despite of a demand for a strong security level. This rigorous requirement in terms of security confines the range of available consensus protocols for public blockchains to the proof-of-work (PoW) protocol. On the contrary, a private blockchain refers to a blockchain platform for identified users, organizations, and entities only. One of the advantages of this platform is to enable equipping of power-efficient consensus protocols instead of resource-consuming consensus protocols due to the strict membership rules of private blockchains. Next, we will introduce Hyperledger Fabric (HLF), which is one of the most popular private blockchain platforms.

\section{B. Hyperledger Fabric}

Hyperledger Fabric (HLF) is an open source blockchain platform under the auspices of the Linux Foundation [6]. Fabric is not only to develop a private modular blockchain platform, but also to enable various smart contracts for wide use cases. Unlike general public blockchains, HLF has the unique structure to prohibit copied ledgers from diverging with supporting non-deterministic computer languages [6]. On the other hand, public systems are possible to cause a blockchain fork, prompting the usage of a deterministic programming language [6]. This distinct structure of HLF is closely related to the transaction flow, which is divided into three phases as described below.
1) Endorsing Phase: The endorsing phase is the first step that a transaction proposal enters at the beginning. The peers, which are entitled to endorse requests and involved in this phase, are referred to as endorsing peers (i.e., endorsers). Their roles are described as follows:

- Endorsing peers execute the proposal using the requested smart contract against their local status so that new values and the current key versions are prepared in a read/write set. The peers then respond to the client with simulation results.

- Endorsing peers make sure that each of the local states in all peers is identical while they perform transaction simulation, in order to prohibit ledger divergence. In case that any of the returned results are different from one another, the client discards the responses and refuses to proceed. If they are identical, on the other hand, the client collects them as a transaction, and transmits it to the ordering service.

2) Ordering Phase: The ordering phase refers to a step in which every endorsed transaction is ordered chronologically. The ordering task is conducted per channel independently in a node cluster. Note that HLF provides the channel concept, in which a channel-specific ledger is only shared across the peers belonging to the same channel for data isolation and confidentiality. Empowered to amass newly generated transactions and to create blocks per channel, the nodes inside the cluster are generally called ordering nodes (i.e., orderers). The new block is delivered to all the peers in the corresponding channel. Contrary to the other components, the ordering service does not appertain to any organization to independently fulfill the 
task.

The ordering service is classified under three implementations according to its internal consensus method: Kafka/ZooKeeper, Byzantine fault tolerant state machine replication (BFT-SMaRt), and RAFT. The Kafka/ZooKeeper is the first fault-tolerant ordering service developed on the basis of Apache Kafka, which is a software platform for highthroughput message management to ensure crash fault tolerance (CFT). The BFT-SMaRt is a Java-based Byzantine fault tolerant protocol for distributed environments, and there is only unofficial version of HLF with BFT-SMaRt, which was launched in [7]. The RAFT ordering service, which is available since 1.4.1v of HLF, is based on a leader-follower model (i.e., RAFT protocol).

3) Validation Phase: The block sent from the ordering cluster enters the last processing step, that is, the validation phase. This step is mainly composed of two sequential verifications: (1) Validation system chaincode (VSCC) verification and (2) Multi-version concurrency control (MVCC) verification. The VSCC verification is to investigate whether the endorsement signature set in the transaction is valid or not. If the set does not satisfy the endorsement policy, the request is not only deemed to be invalid, but also banned from updating the ledger. The MVCC verification is to compare the current versions of the keys captured during the endorsing phase to those in the current states of the ledger, as stored locally by the peer [6]. All data in HLF are stored in the keyvalue scheme, in which each key of data works as a distinct identifier. A change in the version of a key arises in every data update. This principle implies that if both key versions are different, the data was already changed ahead of this transaction. Therefore, the transaction is not only marked as invalid, but also impossible to update the ledger in order to prohibit a blockchain fork [8]. If both versions are equal, on the contrary, the node then writes the new value to its local ledger and commit the block as the latest one.

\section{AoI IN BLOCKCHAIN-ENABLED Networks}

\section{A. Blockchain-enabled Network Structure}

At this point, we state the definition of BCE networks. The BCE network is a particular network in which any service can be provided on the basis of blockchain technology. In other words, any application on the network can be implemented and underpinned based on blockchain in order to furnish users with a specific service.

In Fig. 1, the blockchain platform provider not only facilitates service providers to exploit blockchain for their applications (i.e., authentication history maintenance, temperature detection, and IoT device management), but also helps them to manage and maintain data of their underlying system databases. A new transaction proposal to update the ledger from an external device is transmitted to each assigned endorser to be granted permission. For example, in the IoT device management application network in Fig. 1, an IoT device transmits status data in the form of a transaction proposal to the assigned endorsers. The endorsers simulate the proposal against their own ledgers, then the simulation results are delivered to the IoT device. If the simulation results are identical to each other, the proposal is conveyed to the ordering service in the form of a transaction. The ordering service, where new transactions are assembled, generates a new block per each HLF channel for privacy protection. The new block, which is delivered to the peers, is allowed to update each ledger with the new IoT status data included in the block if the transaction is successfully validated.

\section{B. AoI Elements in Blockchain-enabled Networks}

Expanding the AoI concept to the BCE network requires a different context from traditional networks. The AoI in a traditional network is defined as the elapsed time since the packet for the last update was generated. This definition mainly considers communication latency and queueing-based processing latency. However, in the BCE network, an additional delay, which is not queueing-based, needs to be taken into account because the series of data processing in blockchain also delay updating data. Therefore, we need to consider the time spent in the blockchain (i.e., ledger-commitment latency) as well as the communication latency. One of the main challenges is that real-time applications (e.g., sensor networks, spectrum sharing networks, and vehicle location management network) [1][9] may not be able to provide the latest information for their users due to fast dynamic data. Out-of-date information may incur inaccurate outputs and unintended operations. This problem can be shown clearly in Fig. 1.

In Fig. 1, a sample path of the AoI for IoT status data is illustrated. Since the last update, the AoI is increasing linearly. When a new update occurs at the source at time $t_{g i}$, the new data, which will be committed to the blockchain, takes some time to reach the target blockchain network and becomes effective through the processing steps indicated in Section IIB. The decrease in the AoI at a right angle coincides with the ledger-commitment at time $t_{u i}$. Note that the AoI does not touch zero because the data has the update latency (e.g., $t_{u i^{-}}$ $\left.t_{g i}\right)$ as its AoI until committed to the ledger. For clarification on the proposed topic, we instantiate the elements of AoI in the BCE network in this section.

1) Data Generation Frequency and Time Distribution: The data generation time is closely related to the data update time at the ledger. In the sample AoI path in Fig. 1, when a packet is generated at time $t_{g 1}$ and the block containing the packet is completely committed and updated at time $t_{u 1}$, the AoI of the data in the ledger is reset to $t_{u 1}-t_{g 1}$. The AoI then starts to linearly increase again until the next data update at time $t_{u 2}$. Hence, the AoI depends on two factors: the data generation frequency and the data generation time distribution. The generation frequency is related to the average number of new packets generated per second. The generation distribution can be modelled in a stochastic manner (e.g., exponential distribution) or a deterministic manner (e.g., periodic generation).

2) Communication Latency: The communication latency is the time taken to transmit data to the target blockchain network. The time-stamped packet, which is generated by the source, is conveyed to the associated base station or an access 


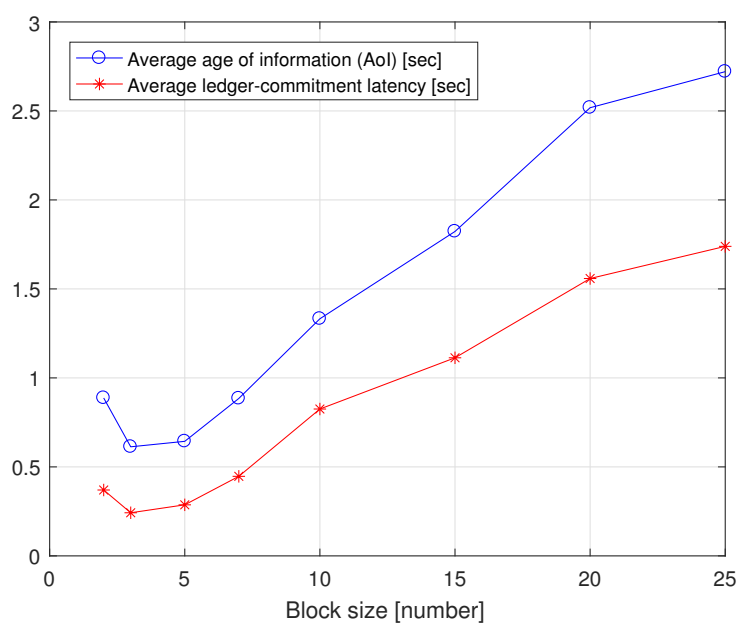

Fig. 2: Effect of the block size on the average AoI and latency, where the block-generation timeout is 2 seconds, and the ratio of the target key transactions is $30 \%$.

point. The data is then forwarded to the BCE network to update the ledger.

3) Ledger-commitment Latency: The ledger-commitment latency refers to the time taken to process a transaction in the BCE network. When the request containing fresh-data successfully arrives at the target blockchain, the data needs to go through the transaction-processing steps, indicated in Section II-B.

From those elements, affecting the AoI, we can also analyze the AoI in various aspects. Once the distribution of the ledgercommitment latency is analyzed (which can be the most challenging part), the average AoI and the complementary cumulative distribution function (CCDF) of AoI can be obtained based on the results in [4] and [5].

\section{INFLUENTIAL FACTOR ANALYSIS}

In this section, we provide experiment results by implementing HLF and show influential factors on the AoI. For the implementation of ordering service, we use Kafka/Zookeeper since it has been most widely used and considered to be more stable than other implementation methods, such as BFT-SMaRt and RAFT. We construct a blockchain platform provider network with all necessary HLF components using HLF 1.3v [10]. Note that the insights obtained in this section can also be applied to HLFs with the other implementation methods of the ordering service since different ordering service only changes how to arrange transactions in a block. The Kafka/ZooKeeper ordering service cluster consists of 4 Kafka nodes with one frontend node. Besides, the blockchain network has one HLF channel, consisting of one endorsing peer and two committing peers, which are only allowed to commit blocks. The request (transaction) arrives at HLF regularly at the rate of 10 transactions/second. A certain percent of the arrived requests tries to update the target key-value. We focus on the AoI of this target data, and this configuration is for all experiments unless specified otherwise.

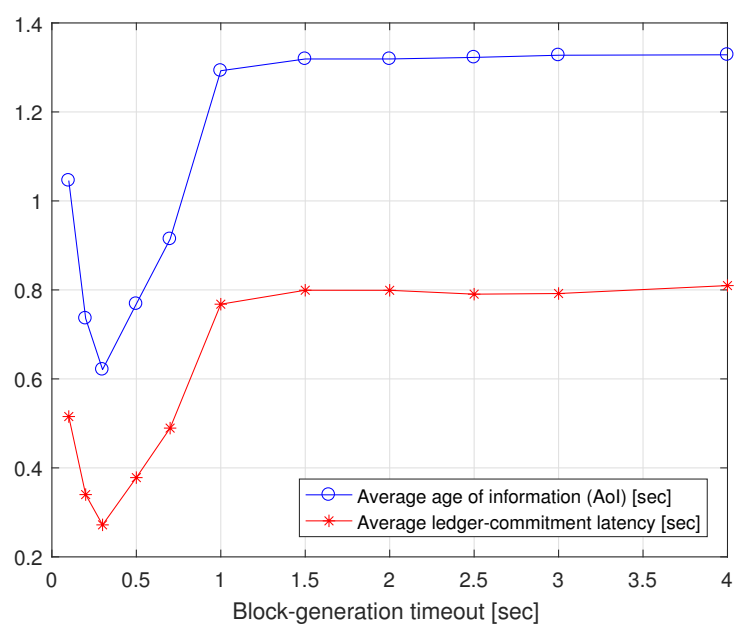

Fig. 3: Effect of the block-generation timeout on the average AoI and latency, where the block size is 10 , and the ratio of the target key transactions is $30 \%$.

\section{A. Blockchain Parameters}

The blockchain parameters refer to blockchain network configuration. In HLF, the block size and the block-generation timeout significantly affect the performance, which we will investigate in the following.

1) Block Size: The block size parameter is the maximum number of transactions in a block. When a transaction arrives in the ordering phase, it is included in a block and awaits others until the number of transactions in the block becomes the block size or the block-generation timeout expires. Consequently, an ordering service with a larger block size results in longer waiting time (i.e., longer ordering latency), as the larger block requires more time to be full. Figure 2 demonstrates the impact of block size. We can see that the average AoI of the target key data increases continuously with the block size. However, when the block size is small (i.e., smaller than three), the AoI decreases with the block size. This unlooked-for result is obtained from the fact that the block generation rate at the ordering service is beyond the block-commitment rate at that point. As a result, new blocks stack up in each peer's waiting queue for commitment. Therefore, it is important to determine appropriate blocksize for shorter AoI in a BCE network.

2) Block-generation Timeout: The block-generation timeout refers to the maximum time that a transaction waits for the others in the ordering service. This parameter is used to avoid long latency by allowing a block to move to the next step even if the block has not been completely full. Hence, as the timeout increases, the blocks are generated slower (i.e., the ordering service latency increases), but the latency in validation phase generally decreases. Figure 3 illustrates the impact of block-generation timeout on the average AoI of the observed data. When the timeout is short (e.g., less than 0.3 seconds), we can see that the average AoI decreases with the timeout. This is because the decrease in the validation latency is more significantly than the increase in the ordering service latency for a short timeout range. On the other hand, 


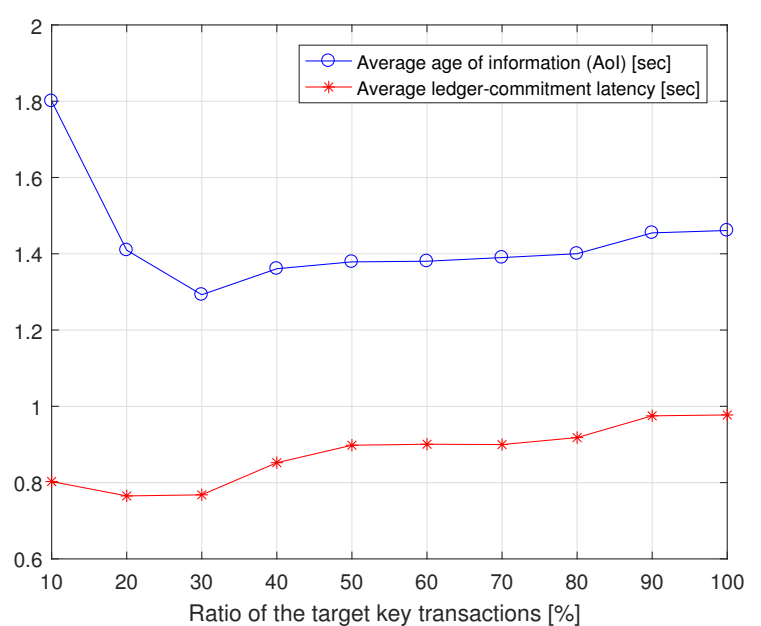

Fig. 4: Effect of the data generation frequency on the average AoI and latency, where the block size is 10 , and the block-generation timeout is 1 second.

when the timeout is greater than 0.3 seconds, the increase in the ordering service latency becomes more significant, so the average AoI increases with the timeout. The average AoI becomes eventually saturated as the timeout becomes large (e.g., greater than 2 seconds) because transactions start to form a compacted block before the timeout expires, which makes the impact of the timeout disappear.

\section{B. Data Generation Frequency}

When data is transmitted through a wireless channel, the other generated data will be on standby to be delivered and cumulate in the transmitter buffer. At this time, it is essential to tune the data generation frequency appropriately since excessively high or low generation frequency has negative influence on the AoI. As another consideration, furthermore, the lastcome first served (LCFS) discipline is worth considering for a packet transmission method than the FCFS discipline. This is because the information of lately generated packet is always fresher than those of packets waiting in the queue. Hence, it is more preferable to transmit the newest packet first than the queued ones through the LCFS discipline. It is also shown that the LCFS scheme can conserve data freshness better than FCFS discipline even at high arrival rate of packets in [11].

Figure 4 shows the impact of ratio of the target key requests (i.e., transactions attempting to update the target data) to the total requests. Note that the impact of communication latency is excluded here, and increasing the ratio is equal to increasing the generation frequency of the target key requests. When the ratio is small, although the average ledger-commitment latency is relatively low due to less processing requests in HLF, the AoI is large due to a lack of information to update. As the ratio increases, the AoI also decreases, but the trend morphs into its opposite after 30 percent. This is from an increase in the possibility that the captured version of the target key at the request generation is different from the current version in the

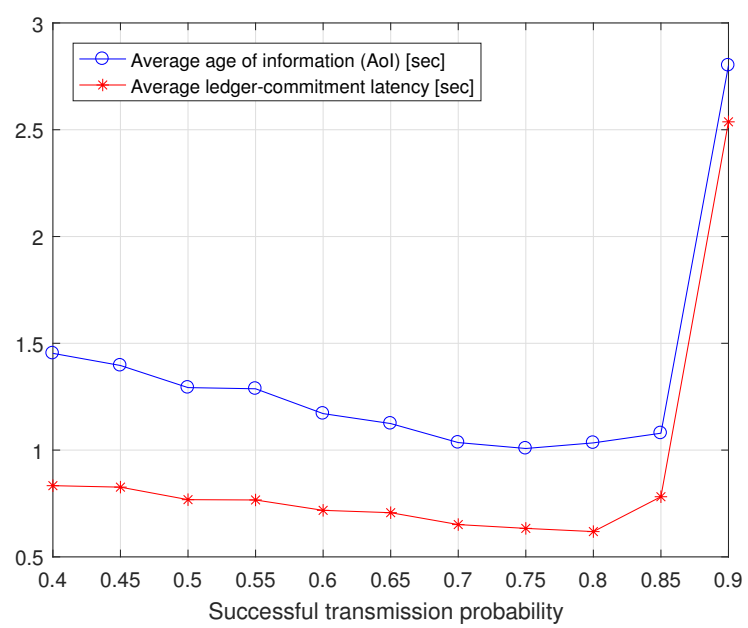

Fig. 5: Effect of the successful transmission probability (STP) on the average AoI and latency, where the total data generation rate is 20 packets/second, the block size is 10, the block-generation timeout is 1 second, and the ratio of the target key transactions is $30 \%$.

ledger at the validation phase, which is considered as invalid during the MVCC verification. This is more likely to occur especially when the key requests generate frequently, since a new request captures the version with which the pre-generated one was already simulated, and the key version can be changed by the previous one before the new request is committed with it. This result not only increases the average latency, but also hinders instantaneous data updates with fresher information.

\section{Communication Parameters}

The communication parameters refer to impactful elements on communication performance, which also affect on the data freshness.

1) Scheduling Policy: The first element we focus on is the scheduling policy, which is referred to a rule to coordinate channel allocation. In a wireless communication system, where multiple nodes can transmit packets, it is necessary for base stations to allocate channels to each node properly. An unsuitable policy may not only incur interference protracting data updates, but also lead to unfair chances to transmit data to update. The biased channel allocation may increase the AoI of particular data in the ledger. Note that the conditions that scheduling policies have to satisfy can change depending on their network environments [12][13] such as the minimum throughput constraint of each node [12]. Therefore, a deliberate selection of scheduling policy must be given to minimize the AoI.

2) Transmission Power: The transmission power of a source is an important element, especially when batteryoperated or energy-constrained sources are deployed [14]. The successful transmission probability (STP), which is defined as the probability that the receiver receives the update data reliably, generally increases with the transmission power of the source. As only successfully received data is used for the information update at the receiver, we expect to have lower 


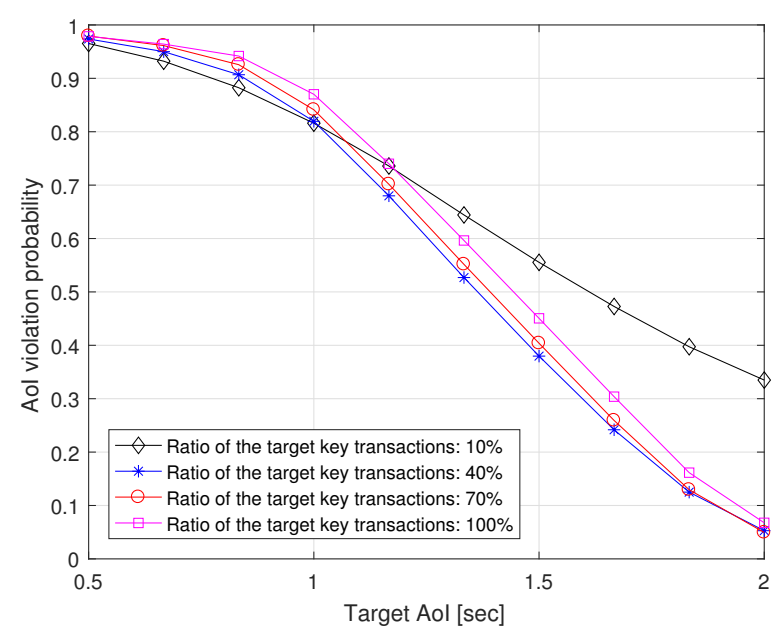

Fig. 6: Effect of the target AoI on the age violation probability, where the block size is 10 , and the block-generation timeout is 1 second.

AoI with higher STP. This result is also shown in Fig. 5, which shows the average AoI with the STP.

However, when the STP is greater than 0.8, the average AoI rapidly increases with the STP. This unforeseen increase is from the long ledger-commitment latency. Specifically, when $\lambda_{\mathrm{t}}$ is the total data generation rate of sources and $\theta$ is the STP, the transaction arrival rate of the HLF channel in the BCE network is defined as $\lambda_{\text {arr }}=\lambda_{t} \theta$. As $\lambda_{\text {arr }}$ increases, blocks are generated faster at the ordering phase, while those blocks need to wait in a queue longer to be committed to the ledger since each block is validated serially in the validation phase. Hence, severely high transaction arrival rate can impede the block-commitment process, as also discussed in Section IV-A.

\section{Future Challenges}

As a prerequisite for successful blockchain utilization, the three influential factors are discussed with experiments in Section IV. We then now need to think about whether blockchain is suitable enough to provide fresh-data requiring services. Let us consider an application that has a target AoI for the system performance. In case of a sensor network, the degree of sensor accuracy decreases with an increase in the AoI of sensing data [15], and the target AoI can be given as the one that guarantees a certain level of sensor accuracy. Figure 6 shows the impact of target AoI on the AoI violation probability, which is the probability that the AoI is greater than the target AoI. When the target AoI is large (e.g., greater than 1.9 seconds), the AoI violation probability is less than 0.1 , which is quite reliable. However, for small target AoI applications, BCE networks may not be suitable. In the rest of this section, we introduce network design aspects that can be considered for data freshness as future challenges as well as possible solutions to lower the AoI from each parameter's perspective.

\section{A. Additional Blockchain Parameters}

Even though the impacts of main blockchain parameters are analyzed in Section IV, the other factors to consider still exist. We cover two additional blockchain considerations in this subsection.

1) Block Generation Rate: The block generation rate refers to the rate of generating a new block at the ordering service. This parameter is not controllable directly, but largely affected by the others blockchain parameters. For instance, generally, the smaller block size, the shorter block-generation timeout, and the higher transaction arrival rate a BCE network has, the new blocks are generated faster. However, as also shown in Figs. 2 and 3, the smallest block size and the shortest timeout do not give the lowest AoI.

When the total arrived transactions during a certain period is $m$ and for $n_{1}<n_{2}$, the time taken to validate and commit $\frac{m}{n_{1}}$ blocks with size $n_{1}$ is not always less than the time taken to validate and commit $\frac{m}{n_{2}}$ blocks with size $n_{2}$, especially for a high transaction arrival rate and a small block size [8]. This is because new blocks flooding from the ordering service into peer buffers at a high rate can lead to longer latency due to the CPU-intensive operations. Therefore, it is important to properly adjust the block generation rate to avoid long latency.

2) Number of Nodes: The AoI can also be affected by the number of participating nodes in blockchain such as endorsing nodes and Kafka nodes of the ordering service in HLF.

- The endorsement policy is a guideline for peers to recognize a properly endorsed transaction [8]. This policy generally specifies by which endorsing peers transactions have to be endorsed. In this sense, users cannot help spending longer time to collect endorsements if multiple endorsing peers are compulsory. In our experiments, it is shown that the average AoI increases from 1.29 to 1.34 seconds as the number of endorsing peers increases from 1 to 3 when the Kafka nodes are four.

- The number of Kafka nodes, used in the ordering service, is connected with CFT, and more Kafka nodes can endure more node failures. For instance, it can allow up to one node failure for four Kafka nodes (i.e., the minimum number) and two node failures for five Kafka nodes. However, as the number of Kafka nodes increases, it requires more processing time. In our experiments, it is shown that the average AoI increases from 1.34 to 1.46 seconds as the number of Kafka nodes increases from 4 to 5 when the endorsing peers are three.

\section{B. Communicational Adjustments}

The optimization of communication systems can effectively reduce the AoI. An optimization method worthy of considering may be to design a novel BCE network-specific scheduling policy, different from the existing frameworks. This policy will perform channel allocation to maintain as much latest information as possible in the ledger.

In Fig. 5, the AoI sharply increases when the STP is over 0.85 . To take precautions against this increase, it is essential to control the high transaction arrival rate of one channel in advance. Inspired by the fact that HLF maintains only one ledger per channel, a multiple ledger policy can be effective to distribute the transaction arrivals over several HLF channels, to eventually reduce the AoI. 


\section{Conclusions}

In this article, we explore whether BCE networks can be used for real-time applications. Utilizing blockchain for application data management induces additional latency, which increases the AoI. However, the blockchain is desired for ensuring data integrity without a trusted third party. Therefore, to maintain fresh data in BCE networks, we discuss how to design influential factors on the AoI in BCE networks including the blockchain parameters, the data generation frequency, and the communication parameters. Specifically, the some of the main insights, obtained in this article, can be summarized as follows:

- The optimal values of the block size, the block-generation timeout, and the STP, which minimize the AoI, exist. This is mainly due to the tradeoff that faster block generation (i.e., smaller block size, shorter timeout, and higher STP) reduces the ordering latency, but increases the validation latency.

- More frequent generation of the update data is generally expected to be better at retaining fresh data. However, it may not be true in the BCE network because frequent transaction generation results in higher probability of being invalid transactions during the MVCC verification.

Eventually, this article provides the initial understanding of BEC networks in the data freshness aspects, and paves the way to reliable BEC networks for AoI-sensitive applications and services.

\section{REFERENCES}

[1] H. Lei and D. Kim, "Design and implementation of an integrated IoT blockchain platform for sensing data integrity," Sensors, vol. 19, no. 10, pp. 1-26, May 2019.

[2] Z. Chen, S. Chen, H. Xu, and B. Hu, "A security authentication scheme of $5 \mathrm{G}$ ultra-dense network based on block chain," IEEE Access, vol. 6, pp. 55 372-55 379, May 2018.

[3] A. Kosta, N. Pappas, and V. Angelakis, "Age of information: A new concept, metric, and tool," Foundations and Trends in Netw., vol. 12, no. 3, pp. 162-259, Nov. 2017.

[4] S. Kaul, R. Yates, and M. Gruteser, "Real-time status: How often should one update?" in Proc. IEEE Int. Conf. on Comput. Commun. (INFOCOM), Orlando, FL, USA, Mar. 2012, pp. 1-5.

[5] Y. Sun, E. U. Biyikoglu, R. Yates, C. E. Koksal, and N. B. Shroff, "Update or wait: How to keep your data fresh," in Proc. IEEE Int. Conf. on Comput. Commun. (INFOCOM), San Francisco, CA, USA, Apr. 2016, pp. 1-9.

[6] E. Androulaki, A. Barger, V. Bortnikov, and C. Cachin, "Hyperledger Fabric: A distributed operating system for permissioned blockchains," in Proc. Europ. Conf. on Comput. Syst. (EuroSys), Porto, Portugal, Jan. 2018, pp. 1-15.

[7] J. Sousa, A. Bessani, and M. Vukolic, "A Byzantine fault-tolerant ordering service for the Hyperledger Fabric blockchain platform," in Proc. IEEE/IFIP Int. Conf. on Dependable Syst. and Netw. (DSN), Luxembourg City, Luxembourg, Jun. 2018, pp. 1-8.

[8] P. Thakkar, S. Nathan, and B. Viswanathan, "Performance benchmarking and optimizing Hyperledger Fabric blockchain platform," in Proc. IEEE Int. Symp. on Modeling, Analysis, and Simulation of Comput. and Telecomm. Syst. (MASCOTS), Milwaukee, WI, USA, Sep. 2018, pp. 113.

[9] M. B. H. Weiss, K. Werbach, D. C. Sicker, and C. E. C. Bastidas, "On the application of blockchains to spectrum management," IEEE Trans. on Cognitive Commun. and Netw., vol. 5, no. 2, pp. 193-205, Apr. 2019.

[10] https://github.com/hyperledger/fabric/releases/tag/v1.3.0.

[11] S. K. Kaul, R. D. Yate, and M. Gruteser, "Status updates through queues," in Proc. Conf. on Inf. Sci. and Syst. (CISS), Princeton, NJ, USA, Mar. 2012, pp. 1-6.
[12] I. Kadota, A. Sinha, and E. Modiano, "Optimizing age of information in wireless networks with throughput constraints," in Proc. IEEE Int. Conf. on Comput. Commun. (INFOCOM), Honolulu, HI, USA, Apr. 2018, pp. $1-9$.

[13] Q. He, D. Yuan, and A. Ephremides, "Optimizing freshness of information: On minimum age link scheduling in wireless systems," in Proc. IEEE Int. Symp. on Modeling and Optim. in Mobile, Ad Hoc, and Wireless Netw. (WiOpt), Tempe, AZ, USA, May 2016, pp. 1-8.

[14] Y. Gu, H. Chen, Y. Zhou, Y. Li, and B. Vucetic, "Timely status update in internet of things monitoring systems: An age-energy tradeoff,' IEEE J. Internet of Things, vol. 6, no. 3, pp. 5324-5335, Jun. 2019.

[15] J. Hribar, M. Costa, N. Kaminski, and L. A. Dasilva, "Using correlated information to extend device lifetime," IEEE J. Internet of Things, vol. 6, no. 2, pp. 2439-2448, Apr. 2018. 